\title{
ARTICLE
}

Received 6 Nov 2013 | Accepted 20 May 2014 | Published 16 Jun $2014 \quad$ DOl: 10.1038/ncomms5171

\section{Stiff filamentous virus translocations through solid-state nanopores}

Angus McMullen ${ }^{1}$, Hendrick W. de Haan ${ }^{2}$, Jay X. Tang ${ }^{1} \&$ Derek Stein ${ }^{1}$

The ionic conductance through a nanometer-sized pore in a membrane changes when a biopolymer slides through it, making nanopores sensitive to single molecules in solution. Their possible use for sequencing has motivated numerous studies on how DNA, a semiflexible polymer, translocates nanopores. Here we study voltage-driven dynamics of the stiff filamentous virus $f d$ with experiments and simulations to investigate the basic physics of polymer translocations. We find that the electric field distribution aligns an approaching $f d$ with the nanopore, promoting its capture, but it also pulls $f d$ sideways against the membrane after failed translocation attempts until thermal fluctuations reorient the virus for translocation. $f d$ is too stiff to translocate in folded configurations. It therefore translocates linearly, exhibiting a voltage-independent mobility and obeying first-passage-time statistics. Surprisingly, lengthwise Brownian motion only partially accounts for the translocation velocity fluctuations. We also observe a voltage-dependent contribution whose origin is only partially determined.

\footnotetext{
${ }^{1}$ Brown University, Department of Physics, Providence, Rhode Island 02912, USA. ${ }^{2}$ University of Ontario Institute of Technology, Faculty of Science, Oshawa, Ontario, Canada L1H 7K4. Correspondence and requests for materials should be addressed to D.S. (email: Derek_Stein@brown.edu).
} 
A nanopore is a nanometer-scale hole in a thin insulating membrane that serves as a sensitive electronic detector. It senses when a single biopolymer threads it by registering a change in its ionic conductance ${ }^{1,2}$. The possibility of applying nanopores to the analysis of nucleic acids, in particular DNA sequencing, has generated interest ${ }^{3}$, and motivated fundamental studies of the physics of nanopore translocations ${ }^{4-17}$. The uses of solid-state nanopores have recently expanded to include detecting single proteins ${ }^{18}$, mapping structural features along RecA-bound DNA-protein complexes ${ }^{19}$ and detecting spherical and icosahedral virus strains ${ }^{20-22}$. Such advances underline the importance of expanding our understanding of nanopore translocations beyond the case of DNA.

A different approach is to study filamentous viruses, an interesting class of DNA-protein complexes with highly controllable physical and chemical properties. Filamentous viruses are also infectious agents that can be important targets for detection, as in the cases of the dangerous Filoviridae Marburg and Ebola ${ }^{23}$. The filamentous virus $f d$ is a bacteriophage that infects Escherichia coli. Its contour length is $L \approx 880 \mathrm{~nm}^{24}$. A protein coat encases the single-stranded DNA genome, giving the virus a roughly circular cross-section with a diameter of $6.6 \mathrm{~nm}^{24}$, and a high negative linear charge density of $-10 e \mathrm{~nm}^{-1}$ at $\mathrm{pH}$ $8.0^{25}$, where $e$ is the elementary charge. The monodispersity of $f d$ makes it ideal for quantitative studies. In addition, $f d$ can be chemically modified, and related virus strains are available with various lengths ${ }^{26}$, charge densities ${ }^{27}$ and stiffnesses ${ }^{28}$.

One can use $f d$ to study the influence of stiffness on a filament's capture by a nanopore and subsequent translocation. The persistence length of $f d$ is $P \approx 2.8 \mu \mathrm{m}^{28}$, which exceeds $L$ by a factor of $\sim 3$; $f d$ therefore behaves like a stiff rod in solution rather than a random coil. By contrast, double-stranded DNA (dsDNA) has a persistence length of only $\sim 50 \mathrm{~nm}^{29}$. It is flexible enough to form hernias at the mouth of a voltage-biased nanopore, and translocate in a folded configuration ${ }^{30}$. Folded configurations are presumably suppressed when $P$ exceeds some threshold.

A polymer's stiffness should also influence its translocation dynamics. According to the conventional theoretical picture, the electrokinetic driving force $\mathrm{e}^{10,12,13}$ and stochastic thermal forces $8,9,15$ are balanced by the total viscous drag on the translocating polymer. The translocation is also retarded by electro-osmotic fluid flow through the nanopore ${ }^{11,31}$. The instantaneous drag includes a large contribution from the length of polymer moving towards the nanopore through solution $^{14-17}$. Consequently, a DNA molecule that first threads the nanopore fully extended will experience more drag and translocate more slowly than if its initial configuration is a tight coil, close to the nanopore. The configuration of a dsDNA coil when it first threads the nanopore therefore determines its average translocation speed, $v^{14,17}$, and the distribution of initial configurations explains fluctuations in $v^{15}$. Fluctuations in $v$ are an impediment to using nanopores to size or sequence DNA.

Here, we present experimental studies and simulations of the capture and translocation of $f d$ in solid-state nanopores. To our knowledge, this is the first report of the translocation of stiff filaments of well-defined shape and size. We are able to distinguish $f d$ 's translocations and blunt collisions with the nanopore from the ionic current signal. The collisions, which are failed translocation attempts by a filament that is too stiff to enter the nanopore in a folded configuration, reveal a competition between electric and thermal forces in the capture process. Analysis of the translocations enables us to test theoretical models in an unexplored regime of polymer stiffness. Since $f d$ is too stiff to coil, we expected its initial configurations and hence its mean translocation velocity to be more uniform than those of DNA. We expected the main source of velocity fluctuations to be the lengthwise Brownian motion of $f d$. We find that the dispersion in $v$ has two causes: translational Brownian motion of the stiff rod along its principal axis and a contribution that grows with voltage, whose possible origin we investigate with simulations and then discuss.

\section{Results}

Ionic current signatures of $\boldsymbol{f} \boldsymbol{d}$-nanopore interactions. The basic principle of our experiments is illustrated in Fig. 1a. A nanopore in a 20 -nm-thick silicon nitride membrane linked two reservoirs of buffered salt solution, across which a voltage, $V$, was applied, resulting in an ionic current through the nanopore, $I$. The filamentous shape and uniform charge of $f d$ suit it well for interrogation by a voltage-biased nanopore, as illustrated in Fig. 1a. Figure $1 \mathrm{~b}$ presents a typical atomic force micrograph of many $f d$ viruses on a mica substrate. The distribution of $L$, obtained from multiple images and presented in Fig. 1c, shows a sharp peak at $L \approx 900 \mathrm{~nm}$. After we introduced $f d$ viruses into the negatively charged reservoir, they were electrophoretically drawn through the nanopore, causing transient changes in $I$.

Figure 2a shows a typical time trace of $I$ from a $26 \mathrm{~nm}$-diameter nanopore, after $f d$ virus was added to the negative reservoir. $V$ was $60 \mathrm{mV}$ and both reservoirs contained $200 \mathrm{mM} \mathrm{KCl}, \mathrm{pH} 8.0$ buffer. The open nanopore current was $6.2 \mathrm{nA}$. Blockages of $I$ are distinguishable from the baseline; several minor blockages are followed by a sustained major blockage $\approx 0.12 \mathrm{nA}$ in amplitude.

Figure $2 \mathrm{~b}$ presents a scatter plot in which each blockage event is represented by a single point whose coordinates are given by the blockage duration, $\tau$, and the mean current change during the blockage, $\left\langle\Delta I_{B}\right\rangle$. (We obtained the instantaneous current change, $\Delta I_{B}$, by subtracting the mean of $4 \mathrm{~ms}$ of current on

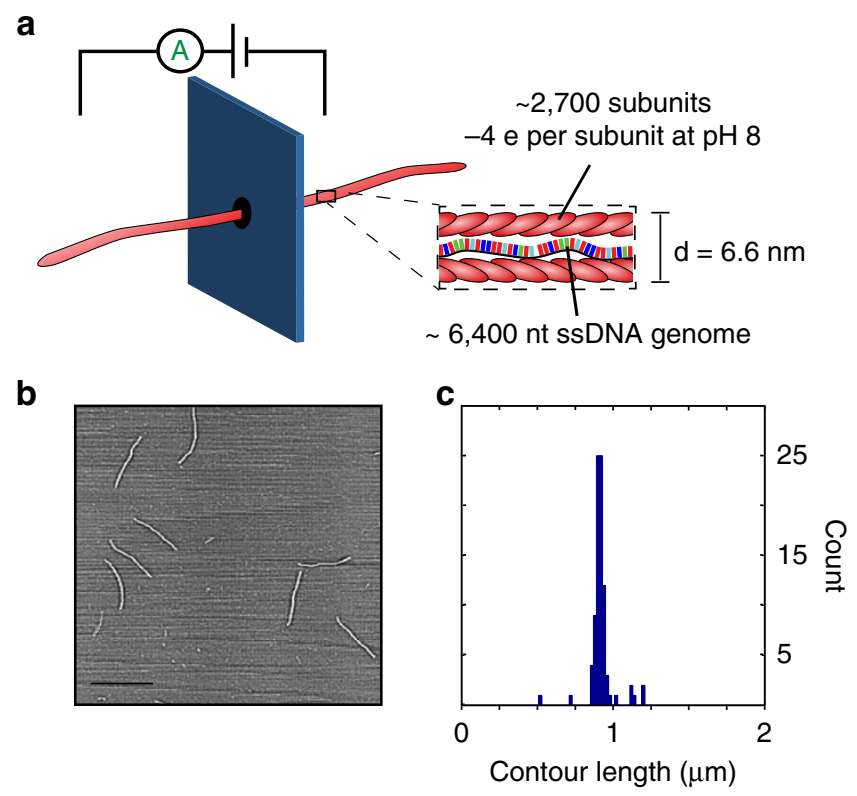

Figure 1 | Studies of filamentous viruses using nanopores. (a) fd virus detection by a solid-state nanopore. The applied voltage draws a virus into the nanopore, where it blocks a measurable amount of ionic current. The detail sketches the single-stranded DNA (ssDNA) in its protein coat, which makes $f d$ virus $880 \mathrm{~nm}$ long and $6.6 \mathrm{~nm}$ wide, with a linear charge density of $-10 \mathrm{enm}^{-1}$ at $\mathrm{pH}$ 8.0. (b) AFM contact mode image of $f d$ virus dried on a mica surface. Scale bar, $1 \mu \mathrm{m}$. (c) A histogram of $f d$ virus lengths $(N=87)$ as measured by atomic force microscopy. 


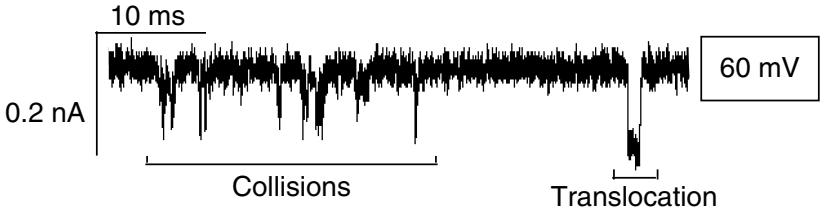

C

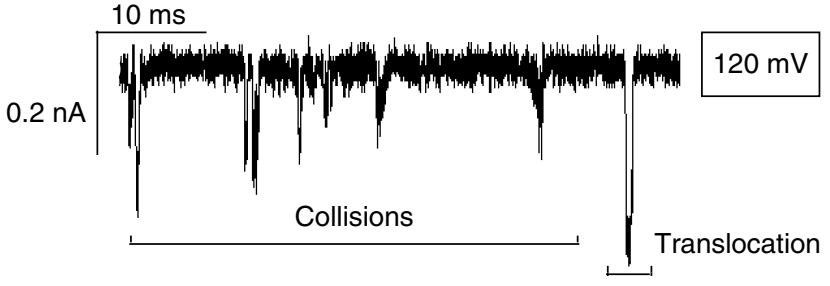

b

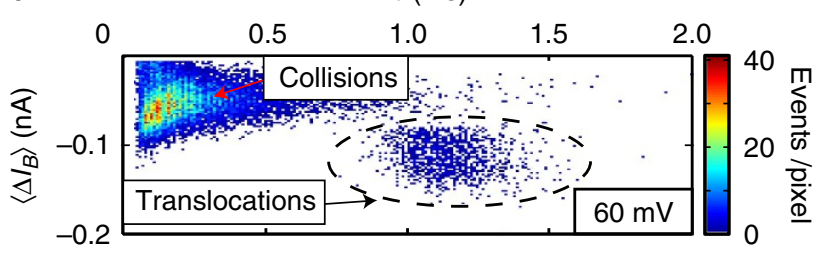

d

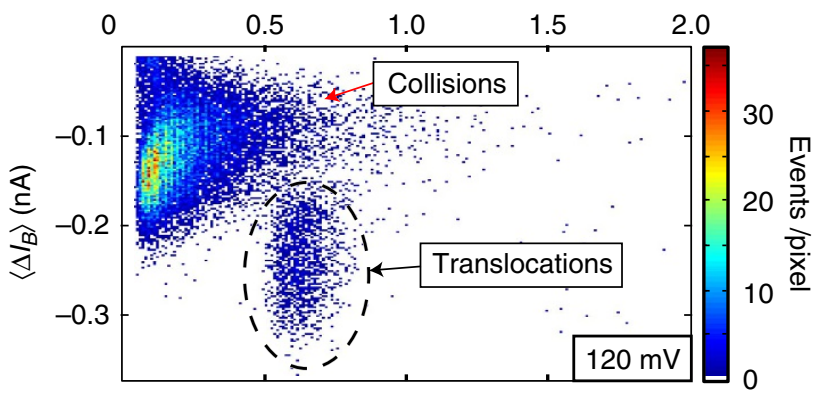

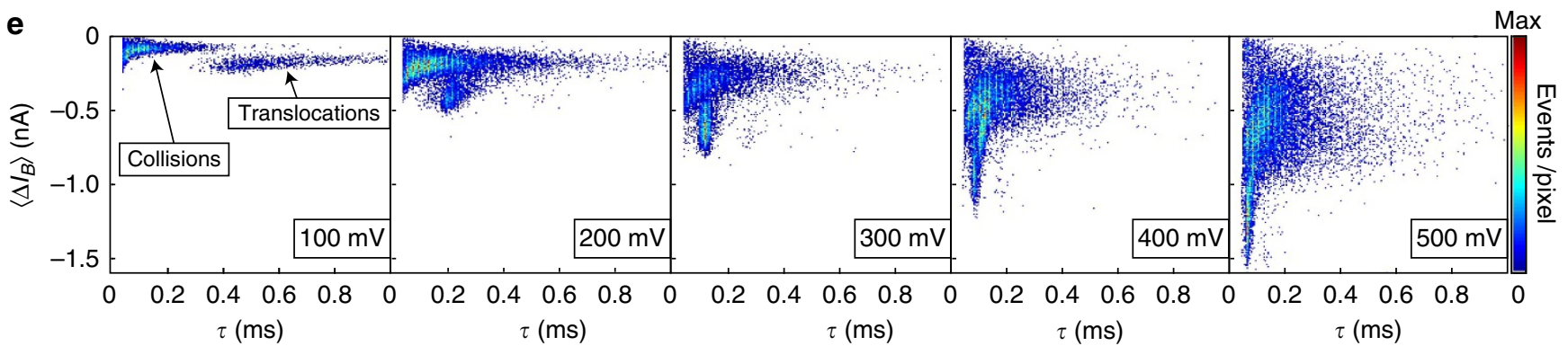

Figure 2 | Electrical signatures of $\boldsymbol{f d}$ interactions with a nanopore. Time traces of $\mathrm{I}$, recorded using a 26 -nm-diameter nanopore, are shown for (a) $60 \mathrm{mV}$ and (c) $120 \mathrm{mV}$. The buffer contained $200 \mathrm{mM} \mathrm{KCl}, 1 \mathrm{mM}$ EDTA and $10 \mathrm{mM}$ Tris- $\mathrm{HCl}(\mathrm{pH} 8.0)$. Scatter plots show the events recorded at (b) $60 \mathrm{mV}$ (3,565 events) and (d) $120 \mathrm{mV}$ (4,704 events) from the same 26-nm-diameter nanopore. (e) Scatter plots of $\left\langle\Delta I_{B}\right\rangle$ versus $\tau$ for $V$ ranging from 100 to $500 \mathrm{mV}$. The measurements used a $19-\mathrm{nm}$-diameter nanopore in $200 \mathrm{mM} \mathrm{KCl}$.

either side of a blockage event from I.) Two distinct populations of events can be seen. The first is centered around $\left\langle\Delta I_{B}\right\rangle \approx-0.12 \mathrm{nA}$ and $\tau \approx 1.2 \mathrm{~ms} ;$ it includes the major, sustained blockage in Fig. 2a, and ionic current signals similar to it. (More examples of major blockages are presented in Supplementary Fig. 1). The second population has smaller $\left\langle\Delta I_{B}\right\rangle$ amplitudes and much shorter $\tau$; it includes the minor current blockages seen in Fig. 2a. (Our method for assigning each event to a particular population is described in the Methods.)

Figure $2 \mathrm{c}$ shows a typical time trace obtained after raising the voltage to $V=120 \mathrm{mV}$. Several minor blockages are again followed by a sustained major blockage. Figure $2 \mathrm{~d}$ displays a scatter plot of the events observed with $V=120 \mathrm{mV}$. Increasing $V$ shifted the first population towards higher $\left\langle\Delta I_{B}\right\rangle$ amplitude and lower $\tau$; doubling $V$ approximately doubled $\left\langle\Delta I_{B}\right\rangle$ and halved $\tau$. The characteristic $\tau$ of the second population depended weakly on $V$, whereas its $\left\langle\Delta I_{B}\right\rangle$ amplitude increased with $V$. Increasing $V$ above $200 \mathrm{mV}$ caused the two populations to overlap on a scatter plot, making them difficult to isolate. Scatter plots of events recorded with $V$ ranging from 100 to $500 \mathrm{mV}$ are presented in Fig. 2e. Events of each type tended to increase in frequency with $V$.

Both populations shifted towards larger $\left\langle\Delta I_{B}\right\rangle$ amplitudes with increasing ionic strength, roughly in proportion to the latter. (A comparison of events recorded at $200 \mathrm{mM} \mathrm{KCl}$ and $500 \mathrm{mM} \mathrm{KCl}$ is presented in Supplementary Fig. 2). Increasing the ionic strength to $300 \mathrm{mM}$ or higher caused the two populations to significantly overlap. Such high salt concentrations also significantly increased the frequency of extremely long blockages that we attribute to $f d$ clogging the nanopore.

We deduce from multiple lines of evidence that the first population of events corresponds to translocations of the nanopore by $f d$. First, the regular time course of the events in that population was consistent with the insertion of a rod of uniform cross-section into the nanopore; those current traces were characterized by an abrupt, flat-bottomed blockage- a shape similar to the well-established signatures of linear DNA translocations ${ }^{7}$. Second, $\tau^{-1}$ and $\left\langle\Delta I_{B}\right\rangle$ were proportional to $V$, as expected for electrophoretically driven translocations. Third, $\left\langle\Delta I_{B}\right\rangle$ was approximately proportional to the salt concentration and its magnitude was in basic agreement with the theoretical model of Smeets et al. ${ }^{4}$, given the known diameter and linear charge density of $f d$ (see Supplementary Note 1). Fourth, the range of $\tau$ was reasonable for $f d$ translocations.

We attribute the second population of events to side-on collisions of the virus with the nanopore. The minor blockage events in that population were characterized by lower $\left\langle\Delta I_{B}\right\rangle$ and $\tau$ than translocations. We expect blunt collisions to block a smaller fraction of $I$ than a virus that inserts fully into the nanopore. We frequently observed minor blockage events occurring in rapid succession before a translocation (see Supplementary Fig. 3). We interpret this pattern as a virus unsuccessfully attempting to translocate before thermal fluctuations present one of its ends to the nanopore and allow it to translocate. Such failed translocation attempts were observed in simulations, as we will discuss later. 


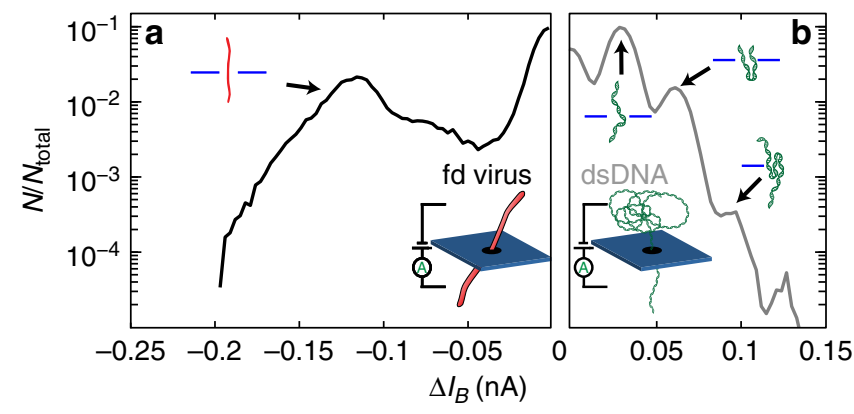

Figure 3 | lonic current change caused by $f d$ and dsDNA translocations. Histograms of the number of $4-\mu$ s-long samples of $\Delta l_{B}$ per $3 p A$ bin $(N)$, normalized by the total number of samples $\left(N_{\text {total }}\right)$, for $f d$ (panel (a), black, 280 translocations) and $\lambda$ DNA (panel (b), grey, 1,434 translocations). The current recordings were performed on a 19-nm-diameter nanopore with $V=100 \mathrm{mV}$ in $100 \mathrm{mM} \mathrm{KCl}$. The histograms represent currents recorded during translocation events and the $100 \mu$ s of baseline that preceded and followed each event. In $100 \mathrm{mM} \mathrm{KCl}$, fd virus translocations caused the current to decrease, whereas $\lambda$ DNA caused it to increase. Positive (negative) values of $\Delta I_{B}$ due to baseline noise are omitted from the $f d(\lambda$ DNA) histogram for clarity.

Translocations through the nanopore. Figure 3 compares histograms of 4- $\mu \mathrm{s}$-long samples of $\Delta I_{B}$ from translocations of the same 19-nm-wide nanopore by $f d$ (black), and by $L=16.5 \mu \mathrm{m}$, double-stranded $\lambda$ DNA (grey), recorded in separate experiments. $V$ was $100 \mathrm{mV}$. The electrolyte contained $100 \mathrm{mM} \mathrm{KCl}$, a concentration at which $f d$ and DNA translocations both produced observable ionic current changes. The DNA histogram reveals a series of approximately equally spaced blockage peaks at positive values of $\Delta I_{B}$, specifically $29 \pm 0.01,61 \pm 0.04,95 \pm 0.5$ (a shoulder rather than a peak) and $125 \pm 0.7 \mathrm{pA}$. The $f d$ histogram reveals a single major blockage peak at $\Delta I_{B} \approx-115 \pm 0.2 \mathrm{pA}$.

Figure 4 a shows the distributions of $f d$ translocation times for $V=60$ and $V=120 \mathrm{mV}$, from the 26-nm-wide nanopore considered in Fig. 2. We quantified the translocation dynamics of $f d$ with the one-dimensional Fokker-Planck equation, which yields the first-passage-time distribution ${ }^{32,33}$

$$
F(\tau)=\frac{L}{\sqrt{4 \pi D_{\|} \tau^{3 / 2}}} \exp \left(-\frac{(L-v \tau)^{2}}{4 D_{\|} \tau}\right) .
$$

We fit equation (1) to the measured $\tau$ distributions using $v$ and $D_{\|}$as fitting parameters. $F(\tau)$ is the probability density for a onedimensional walker with drift velocity $v$ and dispersivity $D_{\|}$to travel a distance $L$ for the first time in an interval $\tau$. Good fits of equation (1) to the data were obtained for all $V$. The dependence of $v$ on $V$, plotted in the inset of Fig. 4a, is well fit by a line passing through the origin. The slope of the line obtains the mobility of $f d$ in the nanopore, $\mu_{f d \text {,pore }}=1.2 \pm 0.1 \mathrm{~cm} \mathrm{~V}^{-1} \mathrm{~s}^{-1}$. The mean mobility in the eight nanopores tested at $200 \mathrm{mM} \mathrm{KCl}$ was $\left\langle\mu_{f d \text {,pore }}\right\rangle=1.4 \pm 0.4 \mathrm{~cm} \mathrm{~V}^{-1} \mathrm{~s}^{-1}$.

The widths of the $\tau$ distributions arise from fluctuations of the translocation velocity, which we quantify with the fit parameter $D_{\|}$. The voltage-dependence of $D_{\|}$is plotted in Fig. $4 \mathrm{~b}$ for eight nanopores. For comparison, the figure also shows the diffusion coefficient of $f d$ for lengthwise motion, $D_{0, \|}=3.5 \mu \mathrm{m}^{2} \mathrm{~s}^{-1}$ (see Methods).

The measured values of $D_{\|}$were typically close to $D_{0, \|}$ for $V \leq 60 \mathrm{mV}$. $D_{\|}$began increasing with $V$ beyond about $60 \mathrm{mV}$. By $V=120 \mathrm{mV}, D_{\|}$exceeded $D_{0, \|}$ by a factor of $2-4$. The rise in $D_{\|}$ was accompanied by a widening spread in values measured on different nanopores. We did not find a clear relationship between
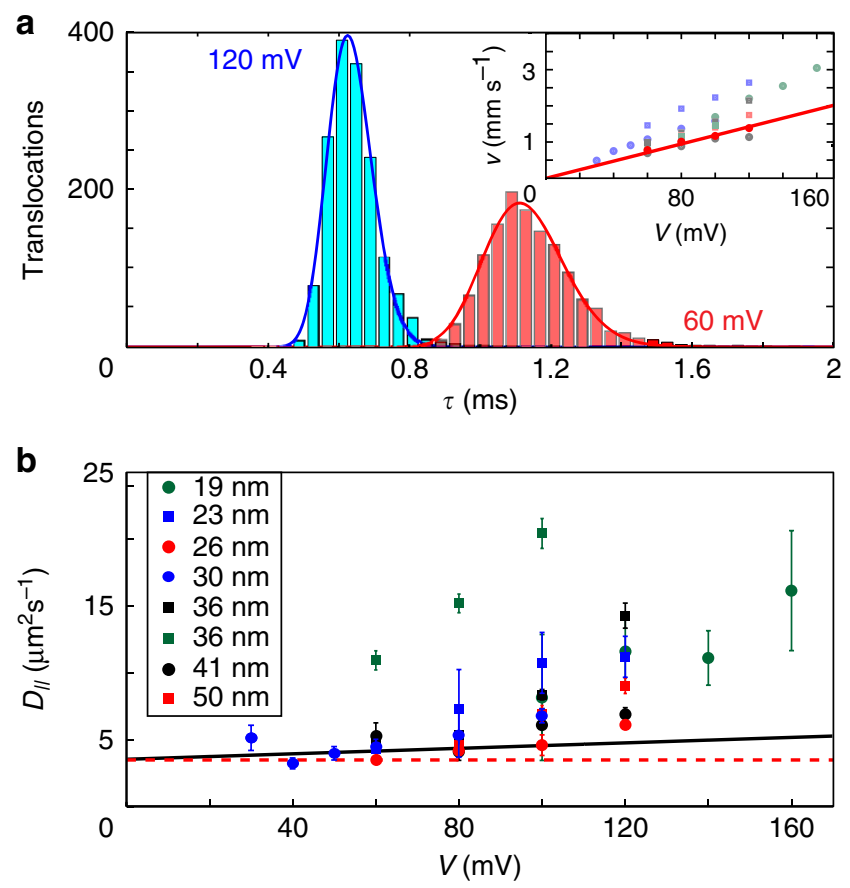

Figure 4 | fd translocation dynamics. (a) Distributions of $\tau$ for $f d$ translocations of a 26-nm-diameter nanopore for $V=120 \mathrm{mV}$ (blue, 1,526 translocations) and $V=60 \mathrm{mV}$ (red, 1,223 translocations). The buffer contained $200 \mathrm{mM} \mathrm{KCl}$. Solid lines are fits of equation (1). Inset: the dependence of $v$ on $V$. S.d. are comparable to the size of the symbols. The line is a linear fit to the data set represented in the histograms, fixed at the origin. (b) Dependence of $D_{\|}$on $V$, from eight nanopores whose diameters are indicated. Error bars show s.d. found by bootstrap resampling of the data. The red dashed line shows $D_{0, \|}$, the lengthwise diffusivity of $f d$ in bulk solution. The black line plots the dependence of $D_{\|}$on $V$ obtained from simulations.

$D_{\|}$and the size of the nanopore. In all nine nanopores with diameters under $15 \mathrm{~nm}$ that we tested with $f d$, the virus repeatedly stuck inside the nanopore, making it difficult to observe a significant number of translocations. We also found no difference in the behaviour of $D_{\|}$, within experimental error, between $f d$ 's translocations and those of an even stiffer mutant, $f d$-Y21M (see Supplementary Fig. 4; Supplementary Note 2).

We performed simulations to investigate possible sources of translocation velocity fluctuations, including the incident angle of the virus on first entering the nanopore, the influence of the electrophoretic forces acting on the virus outside the nanopore and the bending of the finitely deformable virus. Our simulations used coarse-grained Langevin Dynamics ${ }^{41}$. We modelled $f d$ as a generic linear polymer composed of 133 beads of diameter $\sigma$, giving the correct aspect ratio. We imposed a persistence length of $P=423 \sigma$, such that $P=3.2 L$, as with $f d$. We solved the electric potential via a finite difference approach for the nanopore geometry to account for the electric forces inside and outside the nanopore. We simulated different driving forces by multiplying the potential distribution by a constant scaling factor, $\lambda$. We matched simulation and experimental forces by matching the balance between drift and translational diffusion, given by the Péclet number for lengthwise motion, $P e ́=\frac{L^{2}}{\tau D_{0, \|}}$. $P e ́$ is a convenient and meaningful measure of the driving force in simulations $^{42}$. In our simulations, $D_{0, \|}=D_{0}$ (the diffusivity and the lengthwise diffusivity are equal), because hydrodynamic interactions are ignored. See Methods for further details. 
Figure 5a presents sample images from the simulations. The upper image shows a simulated polymer translocating the nanopore; the lower one shows a simulated polymer colliding with the nanopore side-on. Its high stiffness prevented it from forming a hernia and translocating in a folded configuration. The background colours indicate the potential distribution. It has a large gradient outside the nanopore, which draws in the simulated polymer. The force gradients also exert a torque on the simulated polymer that tends to align it with the nanopore.

Equation (1) was fit to distributions of $\tau$ obtained from simulations. The resulting $v$ was proportional to the simulated force and $P e ́$, to a very good approximation. Figure 5b compares distributions of $\tau$ from simulations and an experiment with similar values of Pé. $\tau$ was normalized by the corresponding $L / v$ in each distribution. The simulation distribution is sharper than the experimental one, but similar in other respects. Our fits of equation (1) to the simulation distributions also revealed that $D_{\|}$ increased with the driving field. Figure $5 \mathrm{c}$ plots the fractional enhancement of $D_{\|}$above $D_{0},\left(D_{\|}-D_{0}\right) / D_{0}$, as a function of Pé. The increase in $\left(D_{\|}-D_{0}\right) / D_{0}$ with Pé is well fit by a line passing through the origin.

We make a quantitative comparison between simulations and experiments in Fig. 4b. We fit a line to the simulation results in Fig. $5 \mathrm{c}$, set $D_{0, \|}\left(=D_{0}\right.$ in simulations) to $3.5 \mu \mathrm{m}^{2} \mathrm{~s}^{-1}$ and converted $P e ́$ to $V$ using $P e ́=\left(L\left\langle\mu_{f d \text {,pore }}\right\rangle / D_{0, \|}\right) V$. We found that $V=(48.7 \pm 9.0 \mathrm{mV}) \times \lambda$, (see Methods). Figure $4 \mathrm{~b}$ plots the resulting relationship between $D_{\|}$and $V$ (black line), extrapolated from simulations. Compared with experiments, simulations showed a much slower rise in $D_{\|}$with $V$.

Collisions with the nanopore. Our ability to separate $f d$ 's translocations through the nanopore from its collisions against it
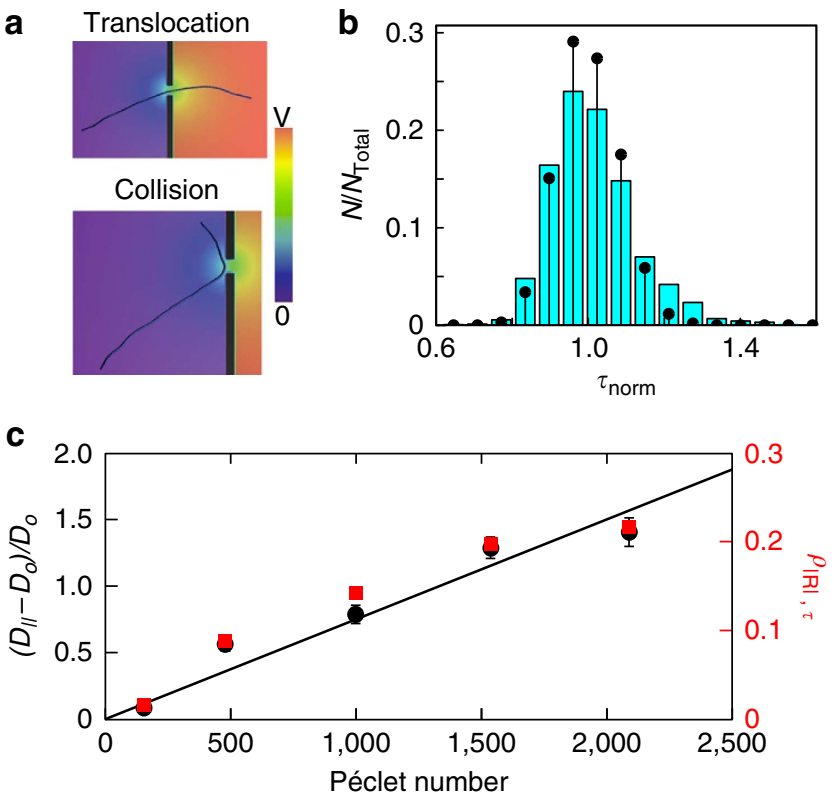

Figure 5 | Simulations of $\boldsymbol{f d}$ translocations. (a) Examples of a translocation (top of panel) and a collision (bottom of panel). The background colour maps the electric potential distribution. (b) Distributions of $\tau$, normalized by $L / v$. Black stems represent 3,751 simulated translocations with $P e ́ \approx 480$. Cyan bars represent 1,526 experimental fd translocations of a 26-nm-diameter nanopore with $V=120 \mathrm{mV}$, for which Pé $\approx 357$. (c) Dependence of $\left(D_{\|}-D_{0}\right) / D_{0}$ (black) and $\rho_{|\mathbf{R}|, \tau}$ (red) on Pé. Error bars show s.d. obtained by bootstrap resampling of the data. The black line is a linear fit, fixed at the origin, to $\left(D_{\|}-D_{0}\right) / D_{0}$ as a function of Pé. enables us to investigate the capture process. We are particularly interested in the efficiency with which the nanopore captures a virus by its end, which is not much smaller than the nanopore itself, relative to failed translocation attempts. It is difficult to count translocation attempts, however, because the result depends on how one distinguishes or groups collision events that occur in rapid succession. Furthermore, we do not know whether two translocation attempts were by the same virus. To avoid these difficulties, we quantify collisions and translocations by their event charge deficit (ECD). ECD is the change in ionic current caused by an event, integrated over the duration of that event ${ }^{6}$. The total ECD of all collisions, $\sum \mathrm{ECD}_{\mathrm{Col}}$, is a useful measure of translocation attempts, and can be compared with the total ECD of all translocations, $\sum \mathrm{ECD}_{\text {Trans. }}$.

Figure $6 \mathrm{a}$ plots the dependence of the ratio $\sum \mathrm{ECD}_{\mathrm{Col}} / \sum$ $\mathrm{ECD}_{\text {Trans }}$ on $V$. The ratio increased with $V$. For the lowest applied
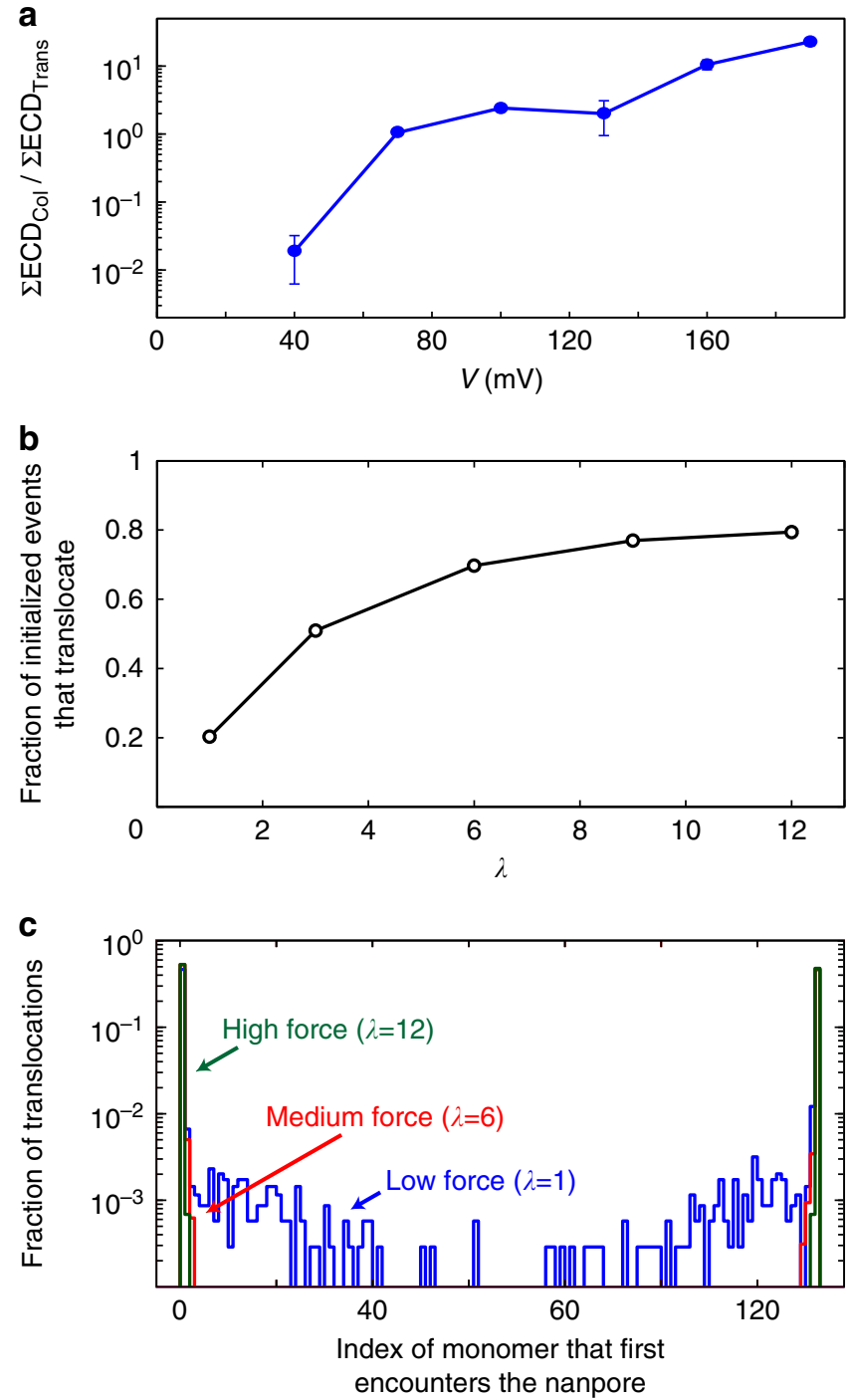

Figure 6 | Translocation attempts. (a) The ratio of the total ECD from collisions to the total ECD from translocations plotted as a function of $V$, from a representative experiment on a 28-nm-diameter nanopore in $200 \mathrm{mM} \mathrm{KCl}$. Errors were calculated by bootstrap resampling. (b) The fraction of initialized simulations that ended with a successful translocation as a function of $\lambda$. (c) Histograms of the index of the first monomer to encounter the nanopore for successful translocations with $\lambda=1$ (blue), $\lambda=6$ (red) and $\lambda=12$ (green). The simulated polymer was 133 monomers long. 
voltages, $\sum \mathrm{ECD}_{\mathrm{Col}}$ was significantly smaller than $\sum \mathrm{ECD}_{\text {Trans }}$. At the high end of the applied voltage range, the situation was reversed, and collisions were the dominant source of ECD.

We also investigated the capture efficiency with simulations. Figure $6 \mathrm{~b}$ plots the fraction of simulated polymers that translocated successfully within the allotted $\approx 8$-ms window against $\lambda$, the simulation force-scaling parameter. The fraction of captures increased with $\lambda$. Figure $6 c$ shows histograms of the index of the first monomer of the simulated polymer to encounter the nanopore for each translocation, for three different values of $\lambda$. A monomer was judged to have encountered the nanopore if it came within $2.5 \sigma$ of the center of the nanopore. For high and medium $\lambda$, successful translocations only occurred if the simulated polymer approached the nanopore end-on (see also Supplementary Fig. 5; Supplementary Fig. 6). At low $\lambda$, on the other hand, the simulated polymer frequently approached the nanopore side-on, had a monomer far from its ends collide with the nanopore, but was still able to reorient and translocate within the time window.

Magnitude of the ionic current changes. The peaks in the experimental $\Delta I_{B}$ histograms (Fig. 3) report the relative frequencies of folded configurations of the translocating filaments. The high intensity at $\Delta I_{B}=0 \mathrm{pA}$ in both histograms corresponds to current through the open nanopore. The first peak in the DNA histogram is caused by the presence of a single segment of DNA inside the nanopore. The second, third and fourth peaks correspond to DNA folded once, twice and three times, respectively, because each additional strand occupying the nanopore causes an incremental change in $I$. Similarly, the peak in the $f d$ histogram at $\Delta I_{B} \approx-115 \mathrm{pA}$ is due to linear translocations. The absence of an additional peak at $\Delta I_{B} \approx-230 \mathrm{pA}$ indicates that $f d$ never translocated in a folded configuration.

The sign and magnitude of $\Delta I_{B}$ are determined by two competing effects: an enhancement of the current carried by counterions along the charged filament, and a reduction in the current carried by bulk ions proportional to the conductivity of the electrolyte and the cross-sectional area of the filament ${ }^{4}$. Both effects are proportional to $V$. Translocations are expected to cause current blockages at high ionic strength, and current enhancements at low ionic strength, with a crossover at $\approx 370 \mathrm{mM} \mathrm{KCl}$ for dsDNA ${ }^{4}$. Accordingly, we observed a current enhancement of $\Delta I_{B} \approx 29 \mathrm{pA}$ for dsDNA at $100 \mathrm{mM}$ $\mathrm{KCl}$. In the case of $f d$, the viral cross-section exceeds that of dsDNA by a factor of $\sim 9$, strongly favouring current blockages, despite its high linear charge density, which is $\sim 1.7$ times that of dsDNA. These properties lead to the rough estimate of a crossover from current blockages to enhancements at $70 \mathrm{mM} \mathrm{KCl}$ for $f d$, in agreement with our observation of current blockage at $100 \mathrm{mM} \mathrm{KCl}$. The theoretical model of Smeets et al. ${ }^{4}$ predicts $\Delta I_{B} \approx-77 \mathrm{pA}$ for dsDNA and $\Delta I_{B} \approx-170 \mathrm{pA}$ for $f d$ (see Supplementary Note 1), whose signs and magnitudes are in reasonable agreement with the measured values for dsDNA $(\approx 29 \mathrm{pA})$ and $f d(\approx-115 \mathrm{pA})$. The discrepancies between theory and experiment are likely traceable to two phenomenological fitting parameters on which that model relies: a Manning condensation parameter that accounts for the reduced mobility of tightly bound counterions, and an effective pore length that accounts for the geometry of the nanopore. Further studies of $f d$ could lead to a better understanding of these important but poorly understood effects.

The translocation velocity. A linear dependence of $v$ on $V$, observed in Fig. 4, is expected for $f d$ translocations. The applied voltage drives the virus with an electrophoretic force and with electro-osmotic flow generated within the charged walls of the nanopore, both of which are linear in $V$. Those driving forces are balanced by Stokes drag, which is linear in $v^{10,11}$.

The mobility of $f d$ is difficult to compute accurately because it depends on the detailed geometry and charge distribution of the nanopore and of the virus. If we instead compare the mobility of $f d$ to that of $\lambda$ DNA translocating under similar conditions $\left(\mu_{\mathrm{DNA}, \text { pore }} \approx 11 \mathrm{~cm} \mathrm{~V}^{-1} \mathrm{~s}^{-1}\right)$, we find that the latter is a factor of 7 higher. We can account for that difference by considering the factors that are theoretically understood to affect the translocation speed. First, we note that the mobility of dsDNA in bulk electrolyte is almost three times higher than for $f d\left(\mu_{\mathrm{DNA}}=3.75\right.$ $\times 10^{-4}$ (ref. 34) versus $\mu_{f d}=1.3 \times 10^{-4} \mathrm{~cm}^{2} \mathrm{~V}^{-1} \mathrm{~s}^{-1}$ (ref. 35), respectively). Second, since silicon nitride nanopores are negatively charged at $\mathrm{pH} 8.0$, translocations of DNA and $f d$ will both be slowed by the same electro-osmotic slip speed, which will amplify the relative difference in their mobilities. Third, the stiff $f d$ virus has a higher viscous drag coefficient than $\lambda$ DNA, and that contributes to the slower translocation speed of $f d$. Viscous drag is felt by whatever part of the polymer is being pulled toward the nanopore through the fluid. That length is different for $f d$ and $\lambda$ DNA. For $\lambda$ DNA, the translocation process is typically fast compared with the relaxation time of the coil. The translocating polymer can therefore be thought of as a dormant portion that experiences no drag, and a moving segment that does $^{36}$. The dormant portion includes the relaxed coil awaiting translocation near the nanopore, and the segments of DNA accumulating on the opposite side after translocating the nanopore. The moving segment is the part under tension as it is drawn towards the nanopore ${ }^{14,15}$. The length of the moving segment is approximately the radius of gyration of the polymer coil, $\approx 730 \mathrm{~nm}$ for $\lambda$ $\mathrm{DNA}^{37}$. (This approximation likely overestimates the characteristic length because it ignores the effect of the trailing end $\left.{ }^{17}\right)$. The diameter of the moving segment is that of the DNA double helix, $2.2 \mathrm{~nm}$, because that segment is under tension. For $f d$, which is much stiffer, the entire contour length is the moving segment and there is no dormant portion on either side of the nanopore. The length and diameter of $f d$ result in a drag coefficient that is about 1.7 times higher than that of the moving segment of $\lambda$ DNA, as calculated for slender rods. In summary, a comparison with DNA shows that the mobility of $f d$ in nanopores is consistent with its distinct physico-chemical properties.

The mobility varied from nanopore to nanopore with a s.d. of $33 \%$, as shown in the inset of Fig. 4a. We speculate that differences in the surface charge of the nanopores partly explain this variation. Differences in the shape of the electric field lines inside the nanopores due to surface roughness or variations in how the nanopore was drilled or cleaned could also explain the spread in mobilities.

Velocity fluctuations. Equation (1) describes the distributions of $f d$ translocation times well (for example, Fig. 4a). That equation presumes a Gaussian distribution of velocity fluctuations, but it makes no assumptions about their physical origin. The lengthwise Brownian motion of $f d$ is a fundamental source of velocity fluctuations that undoubtedly contributes to $D_{\|}$. Nevertheless, it is possible for $D_{\| \mid}$to be lower than $D_{0,|| ;}$ it was recently reported that small, spherical proteins translocating through nanopores exhibited a diffusivity that was voltage-independent and 50 times lower than the bulk diffusion coefficient, a result attributed to confinement and interactions between the protein and the nanopore ${ }^{39}$. $D_{\|}$can also exceed $D_{0, \|}$, as in Taylor-Aris dispersion $^{38}$. We have considered these and other possible sources of dispersion, which are discussed below. 
The lengthwise Brownian motion of $f d$ set a lower bound to the velocity fluctuations. Figure $4 \mathrm{~b}$ shows that $D_{\|}$did not fall below $D_{0}$, within error. $D_{\|}$was typically close to $D_{0, \|}$ for $V \leq 60 \mathrm{mV}$. For $V \geq 100 \mathrm{mV}, D_{\|}$increased beyond $D_{0, \|}$ with $V$. This indicates that there must be a source of velocity fluctuations that is distinct from lengthwise Brownian motion and that depends on the driving force in some fashion.

We used simulations to investigate different mechanisms that could account for this extra contribution to $D_{\|}$. Specifically, we examined the correlations between $\tau$ and the simulated polymer's angular orientation at the onset of translocation, its lateral position inside the nanopore and the flexing of the polymer at the onset of translocation. We found no significant correlation between $\tau$ and the orientation of the simulated polymer, or its lateral position in the nanopore. We found a positive correlation between $\tau$ and the end-to-end distance of the simulated polymer at the onset of translocation, $|\mathbf{R}|$ (see Supplementary Fig. 7). We quantified that correlation by the Pearson product-moment correlation coefficient, $\rho_{|\mathbf{R}|, \tau}$, and plotted its dependence on Pé in Fig. 5c. $\rho_{|\mathbf{R}| \tau}$ increased approximately linearly with Pé. The dependence of $\rho_{|\mathbf{R}|, \tau}$ on Pé was almost identical to that of $\left(D_{\|}-D_{0}\right) / D_{0}$.

The large but finite persistence length allowed the simulated polymer to adopt a variety of bending configurations. A bent configuration at the start of a translocation brings the monomers closer to the nanopore, on average, than an extended configuration. Consequently, the monomers of bent polymers initiate translocations from regions of relatively high force and their short distance to the nanopore results in low fluid drag during translocation. Those two mechanisms explain the decrease in $\tau$ we observed with increasing amounts of initial bending, quantified by $\rho_{|\mathbf{R}|, \tau}$ in simulations. Velocity fluctuations increase with $P$ é because stronger driving forces give diffusion less opportunity to wash out the initial condition of the polymer. The strongest force gave translocation times $(\tau \approx 1,000)$ similar to the simulated polymer's longest relaxation time (637 in simulation units, see Supplementary Fig. 8). When we simulated a polymer with the same high persistence length as the $f d-\mathrm{Y} 21 \mathrm{M}$ strain, we found its $D_{\|}$was lower than that of $f d$ for a given $P e ́$, as expected. The differences in $D_{\|}$were small, however, because $f d$ is very stiff, so its bending configurations are already significantly limited (see Supplementary Fig. 4; Supplementary Note 2). We conclude that the voltage-dependent dispersion in $\tau$ seen in simulations was caused by variations in the initial bending configuration of the simulated polymer.

When compared quantitatively with experiments in Fig. 4b, however, simulations showed a much slower rise in $D_{\|}$with $V$. This indicates that the spread in the viruses' initial bending configurations explains only part of the rise in $D_{\|}$with $V$.

The additional velocity fluctuations of $f d$ at high $V$ must occur by a mechanism not modelled in our simulations. One possibility is that the pulling force depends on the lateral position of the virus inside the nanopore, which drives a mechanism similar to Taylor dispersion ${ }^{38}$. Since $f d$ diffuses laterally inside a nanopore, each virus trajectory would sample the pulling forces differently, leading to additional velocity fluctuations. In a typical translocation, $f d$ diffuses laterally by $60 \mathrm{~nm}$ on average, which is about twice the diameter of the nanopore if we assume $D_{0, \perp}=2.1 \mu \mathrm{m}^{2} \mathrm{~s}^{-1}$ and $\tau=1 \mathrm{~ms}$. The velocity fluctuations should grow with $V$ because fast translocations limit the virus's chance to sample the force landscape. It was recently proposed by Lu et al. ${ }^{12}$ that the net electrokinetic force (on DNA) does in fact depend on the lateral position of the polymer. That detailed electrokinetic force model was not included in our simulations.

Another possibility is the friction between the virus and the nanopore wall. If a virus enters the nanopore obliquely, the electric fields will push the virus against the side of the nanopore and friction will rise. This could create a strong dependence of the translocation time on the angle, with oblique angles expected to generate more friction than if the virus is parallel to the axis of the nanopore. Lower voltages would give more time for rotational diffusion to wash out the effect of the initial angle, leading to a rise in $D_{\|}$with $V$. Our simulations did not include chemical interactions that can generate strong friction. They showed no influence of the angle on the translocation dynamics.

Capture of $f \boldsymbol{d}$ by the nanopore. Our experimental and simulation results paint a coherent picture of the capture of $f d$ by the nanopore. We interpret those results as follows. Figure 6 a shows a rapid growth in collisions relative to translocations as the voltage was raised. Viruses that became trapped sideways by the electric fields were in a metastable state, and required thermal activation to reorient and translocate. Figure $6 \mathrm{a}$ indicates that viruses were drawn ever tighter to the nanopore by the increasing electric fields. This interpretation is also consistent with our observation that collisions increased in frequency with increasing salt concentration (see Supplementary Fig. 2). A higher salt concentration reduced the repulsion between the negatively charged virus and the silicon nitride surface, leading to more collisions. The simulation results in Fig. 6 b show an increasing frequency of successful translocation attempts with voltage. This result reflects the tendency of the electric field gradients to orient an approaching virus towards the nanopore. Experimentally, the rate at which the nanopore captured $f d$ also increased with $V$, partly for the same reason, we expect. Finally, Fig. $6 \mathrm{c}$ shows that successful translocations grow increasingly infrequent with applied force unless the approaching polymer first makes contact with the nanopore near or at its very end. This result illustrates the dual role played by the electric fields, aligning the approaching molecule on one hand, and trapping a molecule after a failed translocation attempt on the other. We note that typical experimental voltages correspond to low values of $\lambda(V=(48.7 \pm 9.0 \mathrm{mV}) \times \lambda$, see Methods $)$. In this regime, the electric forces are less likely to attract the virus to the nanopore end-on, but thermal fluctuations enable it to reorient and translocate after failed attempts.

There is an important qualitative difference between the minor current blockages caused by $f d$ collisions and the minor current blockages recently observed with DNA, which were also interpreted as collisions with the nanopore ${ }^{43-46}$. The DNA collisions reportedly led quickly and reliably to a translocation, whereas $f d$ collisions were not always followed by a translocation. We believe this is because $f d$ is orders of magnitude stiffer than dsDNA and thus forbidden from being captured in a folded configuration, unlike DNA.

\section{Discussion}

We studied the filamentous virus $f d$ to understand the physics of stiff polymer translocations. Our experiments and simulations reveal an interesting interplay between thermal fluctuations and the electric fields outside the nanopore, which determines the capture of $f d$ viruses. The electrophoretic forces tend to align a virus towards the nanopore, facilitating the capture of an approaching virus. If the virus fails to insert its end into the nanopore, however, the same electric fields tend to trap the virus sideways against the nanopore membrane. The electric fields pull the virus harder against the membrane as the applied voltage grows, and it becomes increasingly rare that thermal fluctuations reorient the virus in a way that allows it to translocate. $f d$ was too stiff to enter the nanopore in a folded configuration. The translocation times of $f d$ obey first-passage-time statistics, which are useful for quantifying the mean drift velocity and the 
dispersivity. The velocity of $f d$ is linear in the applied voltage, and consistent with its physical and electrical properties when compared with DNA within the current theoretical picture. The lengthwise Brownian motion of $f d$ is a fundamental source of velocity fluctuations that sets the floor on the value of $f d$ s dispersivity. We discovered an additional, voltage-dependent source of velocity fluctuations that grew increasingly important at high voltages. Langevin dynamics simulations show a correlation between the initial bending configuration and the translocation speed, which grows with voltage. The distribution of initial configurations therefore gives rise to velocity fluctuations, but only a small fraction of what was measured experimentally. There remains an additional source of velocity fluctuations whose origin is not yet properly understood. Finally, we speculate that the labelfree detection of $f d$ might have practical applications, as $f d$ virus is a marker of sewage contamination in ground water ${ }^{47}$, and of $E$. coli contamination of the food supply ${ }^{48}$.

\section{Methods}

Experimental procedures. Nanopores were pierced through 20-nm-thick mem branes made of low-stress LPCVD silicon nitride using a JEOL 2010F or a JEOL $2100 \mathrm{~F}$ high-resolution transmission electron microscope. The detailed fabrication procedure can be found in ref. 49. The transmission electron microscope was also used to measure the diameter of the nanopore. We tested nanopores with diameters ranging from 12 to $50 \mathrm{~nm}$. Each nanopore was cleaned with Nano-Strip (Cyantek) for $2 \mathrm{~h}$ at $75^{\circ} \mathrm{C}$ before use. The nanopore was then mounted in a custom-made fluidic cell.

$f d$ virus was grown and purified according to standard biological procedures ${ }^{50}$, using Xl1-Blue as the host $E$. coli strain. Virus samples were prepared in high concentrations in $100 \mathrm{mM} \mathrm{NaCl}, 20 \mathrm{mM}$ Tris, $\mathrm{pH} 8.0$ buffer, and then diluted to a $0.02 \mathrm{mg} \mathrm{ml}^{-1}(\approx 1 \mathrm{nM})$ virus concentration in the desired buffer solution. The overlap concentration of $f d$ virus is $0.11 \mathrm{mg} \mathrm{ml}^{-1}(\approx 5 \mathrm{nM})^{51}$, so we assume no interaction between $f d$ particles. We prepared samples of similar concentration for the $\lambda$ DNA measurements. These samples were also far below the overlap concentration for a worm-like chain with the same length as $\lambda$ DNA $(48.5 \mathrm{kbp}$ or $16.5 \mu \mathrm{m})^{51}$. Buffer solutions at $\mathrm{pH} 8.0$ were prepared using de-ionized water (Millipore), with $10 \mathrm{mM}$ Tris, $1 \mathrm{mM}$ EDTA and a variable $\mathrm{KCl}$ concentration. The buffer solutions were vortexed for $30 \mathrm{~s}$ to disperse the viruses before adding them to the negative chamber of the fluidic cell.

$\mathrm{Ag}-\mathrm{AgCl}$ electrodes were immersed in both chambers of the fluidic cell and connected to an Axon Axopatch 200B current amplifier, which maintained a constant potential difference while monitoring $I$. The signal was conditioned by an 8-pole Bessel filter with a cutoff frequency of 10 or $50 \mathrm{kHz}$, and then digitized using a $250-\mathrm{kHz}$ sampling rate.

Samples of $f d$ on mica were imaged by atomic force microscopy (AFM) using a Dimension 3100 microscope (Digital Instruments) operating in contact mode. MATLAB was used to threshold the AFM images, measure the contour lengths of the $f d$ filaments and correct for tip-broadening.

Estimation of $\boldsymbol{D}_{\mathbf{0}, \|}$ for $\mathbf{f d}$ virus. We estimated $D_{0, \|}$ from the diffusion coefficient of $f d$ in aqueous buffer at $25^{\circ} \mathrm{C}, D_{0}=2.58 \mu \mathrm{m}^{2} \mathrm{~s}^{-1}$ (ref. 52), the relationship between the parallel and perpendicular components of the diffusion coefficient, $D_{0, \|}$ and $D_{0, \perp}$, respectively, for a rigid rod of finite length and width ${ }^{40}$, and the relationship $D_{0}=\left(2 D_{0, \perp}+D_{0,||}\right) / 3$.

Event extraction and classifications. The analysis of ionic current recordings was performed in two stages using custom MATLAB programs. In the first stage, a program identified the approximate locations of all significant blockage events in the current recordings. In the second stage, a different program determined the precise times of the start and end of each event, and then performed an analysis on the current blockage amplitudes over the course of each event.

The event-identification program stepped a 255-data-point window through the time-ordered current samples point by point, comparing the leading 5 data points $(20 \mu \mathrm{s})$ to the trailing 250 data points $(1 \mathrm{~ms})$, which represent the baseline. When the mean value of the leading section fell below the mean value of the baseline by some threshold amount, the program classified that current fluctuation as an event. The threshold parameter was adjusted depending on the noise characteristics of the current recording. A typical threshold value was 10 times the r.m.s. noise level. A lower threshold was occasionally used when it appeared that translocations were being missed because they were below the threshold, based on a visual inspection of the data. The end of an event was by the mean value of the test section approaching the original baseline level to within $10 \%$ of the r.m.s. noise level. In this manner, the event-identification program quickly scanned the raw data and determined the tentative start and end times of events.
We developed a separate, more computationally intensive program to precisely define the start and end times of events, and to analyse characteristics of those events. That program focused on the events identified in the first stage, plus the 200 data points $(0.8 \mathrm{~ms})$ on either side of each event. The program first smoothed the current using a moving average of five data points, then computed the second derivative of the smoothed current numerically. The precise start and end of an event were defined as the minima occurring within $180 \mu$ s of the tentative start and end times, respectively. (According to this definition, the start of an event corresponds to when the current first leaves the baseline, and the end when it first returns to the baseline). This method determined the start and end times of $f d$ translocations accurately and reliably even in the presence of frequent collision signals, and without employing an arbitrary current threshold. These features were important for obtaining robust measurements of $D_{\|}$across different experimental conditions.

Each event was classified as either a collision or a translocation based on its ECD value ${ }^{6}$. ECD is the integrated current change from the local baseline over the duration of the event. Figure 7 shows a scatter plot of events in terms of $\left\langle\Delta I_{B}\right\rangle$ and $\tau$ from the same $V=60 \mathrm{mV}$ recording presented in Fig. 2. A line indicating $\mathrm{ECD}=-0.07 \mathrm{pC}$ is also plotted in Fig. 7. Events below the line $(|\mathrm{ECD}|>0.07 \mathrm{pC})$ belong to the translocation population, and those above it $(|\mathrm{ECD}|<0.07 \mathrm{pC})$ belong to the collision population. Since collisions tend to be shorter than translocations, it is possible they were attenuated owing to the bandwidth limitations of our electrometer. This could decrease the apparent $|\mathrm{ECD}|$ of collisions. For some experiments, a filter was applied to remove outliers characterized by low $\left\langle\Delta I_{B}\right\rangle$ and high r.m.s. noise levels from the translocation population; we interpret longlived events with low $\left\langle\Delta I_{B}\right\rangle$, which yield a high $|\mathrm{ECD}|$, as the products of unusually long collisions, rather than translocations. An additional cutoff was necessary for some higher voltages $(V \geq 160 \mathrm{mV})$. At these voltages, collisions often appeared in quick succession. The current values just before and after (within $0.1 \mathrm{~ms}$ ) these collisions, or the local baseline, were often lower than the open pore current. Translocations were more likely to have local baselines equal to the open pore current. If an event had a local baseline that was lower than the open pore current, within noise, the event was classified as a collision.

We note here that we have excluded outliers in event duration for Fig. 3 ( $\tau \geq 1 \mathrm{~ms}$ ) to discount any stuck viruses. In addition, we low-pass filtered the current in this data set with an additional $20-\mathrm{kHz}$-low-pass software filter to remove digitization noise, so that we could employ a finer bin size. Since the data were originally collected with a $10-\mathrm{kHz}$-low-pass hardware Bessel filter, this software filter did not affect the appearance of the folding peaks apart from the removal of digitization noise. We also note that all collisions with $|\mathrm{ECD}|$ 's $>6$ s.d. away from the mean $|E C D|$ were excluded from Fig. 6 a to avoid including clogs. The same was done with translocations in Fig. 6a.

Langevin dynamics simulations of $\mathbf{f d}$ translocations. We used a standard coarse-grained approach to model the $f d$ virus as a polymer consisting of 133 monomers of size $\sigma^{41}$. We bound adjacent monomers together by the finitely extensible nonlinear elastic potential:

$$
U_{\mathrm{FENE}}(r)=-\frac{1}{2} k r_{0}^{2} \ln \left(1-\frac{r^{2}}{r_{0}^{2}}\right)
$$

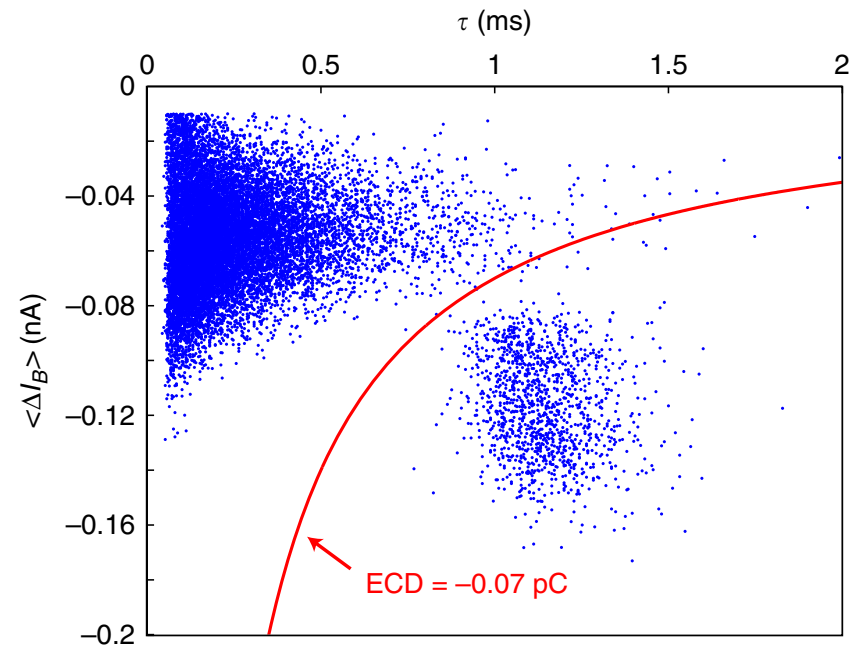

Figure 7 | Separating translocations from collisions. Scatter plot of $\left\langle\Delta I_{B}\right\rangle$ versus $\tau$ for 3,565 events recorded using a 26-nm-diameter nanopore, with $V=60 \mathrm{mV}$, in $200 \mathrm{mM} \mathrm{KCl}, \mathrm{pH} 8.0$ buffer. The red line indicates the constant $\mathrm{ECD}=-0.07 \mathrm{pC}$ value used as the boundary between translocation events and collision events. 
where we set the spring constant $k$ to $30 \epsilon / \sigma^{2}$ (with $\epsilon$ being the characteristic energy) and the maximum extension $r_{0}$ to $1.5 \sigma$ as in the model of Kremer and Grest ${ }^{53}$. A shifted and truncated Lennard-Jones potential often called the WCA potential ${ }^{54}$ gave the excluded volume interactions between monomers:

$$
U_{\mathrm{WCA}}(r)=\left\{\begin{array}{cc}
4 \epsilon\left[\left(\frac{\sigma}{r}\right)^{12}-\left(\frac{\sigma}{r}\right)^{6}\right]+\epsilon & \text { for } r<r_{c} \\
0 & \text { for } r \geq r_{c}
\end{array}\right.
$$

where the cutoff distance $r_{c}$ was set to $2^{1 / 6} \sigma$. We implemented the persistence length by a bending potential given by

$$
U_{\text {bend }}(\theta)=\frac{1}{2} k\left(\theta-\theta_{0}\right)^{2}
$$

where $\theta$ is the angle formed by three consecutive monomers, $k$ is the spring constant and $\theta_{0}$ is equilibrium angle. To model $f d$ virus, we set $\theta_{0}$ to $180^{\circ}$ and $k=423$, yielding a persistence length of $423 \sigma$ and giving the correct ratio of persistence length to contour length.

We used a continuous surface of thickness $3 \sigma$ for the membrane. The diameter of the cylindrical nanopore was $d_{\text {pore }}=5 \sigma$, corresponding to a typical experiment, where $d_{\text {pore }} \approx 30 \mathrm{~nm}$ and $l_{\text {pore }} \approx 20 \mathrm{~nm}$. The details of the potential resulting from applying a voltage drop of $\Delta V$ across this geometry were solved via a finite difference approach.

For computational efficiency, the simulations did not explicitly include solvent. Instead, we used a Langevin Dynamics approach in which the effects of the solvent are included implicitly by including both a damping term and a random force term directly in the equation of motion. The final equation of motion is then given by

$$
m \ddot{\mathbf{r}}=\mathbf{F}_{e x t}-\nabla U(\mathbf{r})-\zeta \dot{\mathbf{r}}+\xi(t)
$$

where $m$ is the monomer mass, $F_{\text {ext }}$ is the external force, $U(\mathbf{r})$ is the sum of the conservative potentials $\left(U_{\mathrm{FENE}}(r), U_{\mathrm{WCA}}(r)\right.$ and $\left.U_{\mathrm{bend}}(\theta)\right), \zeta$ is the friction coefficient and $\xi(t)$ is the random force term. $\xi(t)$ satisfies the following criteria:

$$
\langle\xi(t)\rangle=0
$$$$
\langle\xi(0) \cdot \xi(t)\rangle=2 k_{B} T \zeta \delta(t)
$$

in accordance with the fluctuation-dissipation theorem.

$F_{\text {ext }}$ was adjusted using a scaling parameter, $\lambda$, to change the applied force on the simulated polymer. Simulation forces were matched to experimental conditions by ensuring the balance between drift and diffusion was the same in simulations as in experiments. This balance was calculated via a translocation Péclet number defined as $P e ́=L v / D=\left(\left(L\left\langle\mu_{f d \text {,pore }}\right\rangle\right) /\left(D_{0, \|} \mid\right)\right) V$. We measured the experimental mobility of $f d$ virus translocations to be $\left\langle\mu_{f d \text {,pore }}\right\rangle=14.0 \pm 3.8 \mathrm{~mm} \mathrm{~V}^{-1} \mathrm{~s}^{-1}$. Using the known length of $f d$ virus and its diffusion coefficient, $D_{0, \|}=3.5 \mu \mathrm{m}^{2} \mathrm{~s}^{-1}$, we obtained $P e_{\exp }=(3.52 \pm 0.95) \times 10^{3} V$, where $V$ is the applied voltage in volts. For $V=100 \mathrm{mV}, P e_{\exp }=352 \pm 95$.

We applied a similar method to determine the simulation Péclet number. We measured the simulation mobility to be $\left\langle\mu_{\text {sim }}\right\rangle=(9.7 \pm 0.28) \times 10^{-3}$ a.u. The simulation length, $L$, is $N=133$, while the diffusion coefficient $D_{\text {sim }}=1 / N$. We obtained $P \dot{e}_{\text {sim }}=(172 \pm 05) \lambda$. Matching $P e_{\exp }$ and $P e_{\text {sim }}$, we find that $V=(48.7 \pm 9.0 \mathrm{mV}) \times \lambda$

Simulations were performed using the ESPResSo package ${ }^{55}$ on the SHARCNET computer system (http://www.sharcnet.ca) using visual molecular dynamics ${ }^{56}$ for visualization.

\section{References}

1. Kasianowicz, J. J., Brandin, E., Branton, D. \& Deamer, D. W. Characterization of individual polynucleotide molecules using a membrane channel. Proc. Natl Acad. Sci. USA 93, 13770-13773 (1996).

2. Li, J. et al. Ion-beam sculpting at nanometre length scales. Nature 412, 166-169 (2001).

3. Branton, D. et al. The potential and challenges of nanopore sequencing. Nat. Biotechnol. 26, 1146-1153 (2008)

4. Smeets, R. M. et al. Salt dependence of ion transport and DNA translocation through solid-state nanopores. Nano. Lett. 6, 89-95 (2006).

5. Wanunu, M., Morrison, W., Rabin, Y., Grosberg, A. Y. \& Meller, A. Electrostatic focusing of unlabelled DNA into nanoscale pores using a salt gradient. Nat. Nanotech. 5, 160-165 (2010).

6. Fologea, D., Brandin, E., Uplinger, J., Branton, D. \& Li, J. DNA conformation and base number simultaneously determined in a nanopore. Electrophoresis $\mathbf{2 8}$, 3186-3192 (2007)

7. Li, J., Gershow, M., Stein, D., Brandin, E. \& Golovchenko, J. A. DNA molecules and configurations in a solid-state nanopore microscope. Nat. Mater. 2, 611-615 (2003).

8. Li, J. \& Talaga, D. S. The distribution of DNA translocation times in solid-state nanopores. J. Phys. Condens. Matter. 22, 454129 (2010).

9. Lubensky, D. K. \& Nelson, D. R. Driven polymer translocation through a narrow pore. Biophys. J. 77, 1824-1838 (1999).

10. van Dorp, S., Keyser, U. F., Dekker, N. H., Dekker, C. \& Lemay, S. G. Origin of the electrophoretic force on DNA in solid-state nanopores. Nat. Phys. 5, 347-351 (2009)
11. Ghosal, S. Effect of salt concentration on the electrophoretic speed of a polyelectrolyte through a nanopore. Phys. Rev. Lett. 98, 238104 (2007).

12. Lu, B., Hoogerheide, D., Zhao, Q. \& Yu, D. Effective driving force applied on DNA inside a solid-state nanopore. Phys. Rev. E 86, 011921 (2012).

13. Wong, C. T. \& Muthukumar, M. Polymer capture by electro-osmotic flow of oppositely charged nanopores. J. Chem. Phys. 126, 164903 (2007).

14. Grosberg, A., Nechaev, S., Tamm, M. \& Vasilyev, O. How long does it take to pull an ideal polymer into a small hole? Phys. Rev. Lett. 96, 228105 (2006).

15. Lu, B., Albertorio, F., Hoogerheide, D. P. \& Golovchenko, J. A. Origins and consequences of velocity fluctuations during DNA passage through a nanopore. Biophys. J. 101, 70-79 (2011).

16. Storm, A. J., Chen, J. H., Zandbergen, H. W. \& Dekker, C. Translocation of double-strand DNA through a silicon oxide nanopore. Phys. Rev. E 71, 051903 (2005).

17. Ikonen, T., Bhattacharya, A., Ala-Nissila, T. \& Sung, W. Influence of nonuniversal effects on dynamical scaling in driven polymer translocation. J. Chem. Phys. 137, 085101 (2012).

18. Yusko, E. C. et al. Controlling protein translocation through nanopores with bio-inspired fluid walls. Nat. Nanotech. 6, 253-260 (2011).

19. Smeets, R. M., Kowalczyk, S. W., Hall, A. R., Dekker, N. H. \& Dekker, C. Translocation of RecA-coated double-stranded DNA through solid-state nanopores. Nano Lett. 9, 3089-3095 (2009).

20. Zhou, K., Li, L. \& Tan, Z. Characterization of hepatitis B virus capsids by resistive-pulse sensing. J. Am. Chem. Soc. 133, 1618-1621 (2011).

21. Harms, Z. \& Mogensen, K. Nanofluidic devices with two pores in series for resistive-pulse sensing of single virus capsids. Anal. Chem. 83, 9573-9578 (2011)

22. Uram, J. D., Ke, K., Hunt, A. J. \& Mayer, M. Submicrometer pore-based characterization and quantification of antibody-virus interactions. Small 2, 967-972 (2006).

23. National Institute of Allergy and Infectious Diseases (NIAID). NIAID category $\mathrm{A}, \mathrm{B}$, and C priority pathogens, http://www.niaid.nih.gov/topics/ BiodefenseRelated/Biodefense/Pages/CatA.aspx (2013).

24. Day, L., Marzec, C., Resiberg, S. \& Casadevall, A. DNA packing in filamentous bacteriophages. Ann. Rev. Biophys. Biophys. Chem. 17, 509-539 (1988).

25. Zimmermann, K., Hagedorn, H., Heuck, C. C., Hinrichsen, M. \& Ludwig, H. The ionic properties of the filamentous bacteriophages Pf1 and fd. J. Biol. Chem. 261, 1653-1655 (1986).

26. Dogic, Z. \& Fraden, S. Development of model colloidal liquid crystals and the kinetics of the isotropic-smectic transition. Phil. Trans. R. Soc. Lond. A 359, 997-1015 (2001).

27. Tang, J. X., Janmey, P., Lyubartsev, A. \& Nordenskiold, L. Metal ion-induced lateral aggregation of filamentous viruses fd and M13. Biophys. J. 83, 566-581 (2002).

28. Barry, E., Beller, D. \& Dogic, Z. A model liquid crystalline system based on rodlike viruses with variable chirality and persistence length. Soft Matter 5, 2563-2570 (2009).

29. Bustamante, C., Marko, J., Siggia, E. \& Smith, S. Entropic elasticity of lambdaphage DNA. Science 265, 1599-1600 (1994).

30. Mihovilovic, M., Hagerty, N. \& Stein, D. Statistics of DNA capture by a solidstate nanopore. Phys. Rev. Lett. 110, 028102 (2013).

31. Di Fiori, N. et al. Optoelectronic control of surface charge and translocation dynamics in solid-state nanopores. Nat. Nanotech. 8, 946-951 (2013).

32. Schrödinger, E. Zur theorie der fall-und steigversuche an teilchen mit brownscher bewegung. Phys. Zs. 16, 289-295 (1915).

33. Smoluchowski, M. V. Uber die zeitliche veranderlichkeit der gruppierung von emulsionsteilchen und die reversibilitat der diffusionserscheinungen. Phys. Zs. 16, 321-327 (1915).

34. Stellwagen, N. C., Gelfi, C. \& Righetti, P. G. The free solution mobility of DNA. Biopolymers 42, 687-703 (1997).

35. Wen, Q. \& Tang, J. X. Absence of charge inversion on rodlike polyelectrolytes with excess divalent counterions. J. Chem. Phys. 121, 12666-12670 (2004).

36. Saito, T. \& Sakaue, T. Cis-trans dynamical asymmetry in driven polymer translocation. Phys. Rev. E 88, 042606 (2013).

37. Smith, D. E., Perkins, T. T. \& Chu, S. Dynamic scaling of DNA diffusion coefficients. Macromolecules 29, 1372-1373 (1996).

38. Taylor, G. Dispersion of soluble matter in solvent flowing slowly through a tube. Proc. R. Soc. Lond. A 219, 186-203 (1953).

39. Larkin, J., Henley, R. Y., Muthukumar, M., Rosenstein, J. K. \& Wanunu, M. High-bandwidth protein analysis using solid-state nanopores. Biophys. J. 106, 696-704 (2014).

40. Li, G. \& Tang, J. X. Diffusion of actin filaments within a thin layer between two walls. Phys. Rev. E 69, 061921 (2004)

41. Slater, G. W. et al. Modeling the separation of macromolecules: A review of current computer simulation methods. Electrophoresis 30, 792-818 (2009).

42. de Haan, H. W., Sean, D. \& Slater, G. W. Using a Péclet Number for the Translocation of DNA through a Nanopore to Tune Coarse-Grained Simulations to Experimental Conditions. Preprint at http://arxiv.org/abs/ 1404.4317 (2014). 
43. Kowalczyk, S. W. \& Dekker, C. Measurement of the docking time of a DNA molecule onto a solid-state nanopore. Nano Lett. 12, 4159-4163 (2012).

44. Wanunu, M., Sutin, J., McNally, B., Chow, A. \& Meller, A. DNA translocation governed by interactions with solid-state nanopores. Biophys. J. 95, 4716-4725 (2008).

45. Vlassarev, D. \& Golovchenko, J. A. Trapping DNA near a solid-state nanopore. Biophys. J. 103, 352-356 (2012).

46. Rosenstein, J., Wanunu, M. \& Merchant, C. Integrated nanopore sensing platform with sub-microsecond temporal resolution. Nat. Methods. 9, 487-492 (2012).

47. Martin, C. The application of bacteriophage tracer techniques in south west water. Water Environ. J. 2, 638-642 (1988).

48. Mao, C., Liu, A. \& Cao, B. Virus-based chemical and biological sensing. Angew. Chem. Int. Ed. 2, 638-642 (2009).

49. Jiang, Z., Mihovilovic, M., Teich, E. \& Stein, D. Nanopore-based technology: single molecule characterization and DNA sequencing. (ed Gracheva, M.) (Humana Press, 2009).

50. Sambrook, J., Fritsch, E. F. \& Maniatis, T. Molecular Cloning Vol. 2 (Cold Spring Harbor Laboratory Press, 1989).

51. Ying, Q. \& Chu, B. Overlap concentration of macromolecules in solution. Macromolecules 20, 362-366 (1987).

52. Newman, J., Swinney, H. L. \& Day, L. A. Hydrodynamic properties and structure of fd virus. J. Mol. Biol. 116, 593-603 (1977).

53. Grest, G. S. \& Kremer, K. Molecular dynamics simulation for polymers in the presence of a heat bath. Phys. Rev. A 33, 3628-3631 (1986).

54. Weeks, J. D., Chandler, D. \& Anderson, H. C. Role of repulsive forces in determining the equilibrium structure of simple liquids. J. Chem. Phys. 54, 5237-5247 (1978).

55. Limbach, H. J., Arnold, A., Mann, B. A. \& Holm, C. ESPResSo-an extensible simulation package for research on soft matter systems. Comput. Phys. Commun. 174, 704-727 (2006).
56. Humphrey, W., Dalke, A. \& Schulten, K. VMD: visual molecular dynamics. J. Mol.. Graph. 14, 33-38 (1996).

\section{Acknowledgements}

We thank Zvonimir Dogic, Prerna Sharma and support from NSF DMR—0820492 for virus samples, Guanglai Li for AFM imaging and Sean Ling for bringing to our attention the correct expression for the first-passage-time probability distribution. This work was supported by NSF CBET0846505, NSF PHYS1058375 and the Brown University Institute for Molecular and Nanoscale Innovation.

\section{Author contributions}

A.M.M., J.X.T. and D.S. conceived the project and designed the experiments. A.M.M. performed the experiments and analysed the data. H.W.d.H. designed and performed the simulations. A.M.M., H.W.d.H., J.X.T. and D.S. interpreted the results and wrote the manuscript.

\section{Additional information}

Supplementary Information accompanies this paper at http://www.nature.com/ naturecommunications

Competing financial interests: The authors declare no competing financial interests.

Reprints and permission information is available online at http://npg.nature.com/ reprintsandpermissions/

How to cite this article: McMullen, A. et al. Stiff filamentous virus translocations through solid-state nanopores. Nat. Commun. 5:4171 doi: 10.1038/ncomms5171 (2014). 